\title{
Encapsulation of metal oxide nanoparticles by oxide supports during epitaxial growth
}

Xuan Cheng ${ }^{1,3}$, Jackson Chi Wong ${ }^{1}$, Matthew Weyland ${ }^{2,3}$ and Nagarajan Valanoor ${ }^{{ }^{*}}$

${ }^{1}$ School of Materials Science and Engineering, University of New South Wales, Sydney, NSW 2052, Australia

${ }^{2}$ Monash Centre for Electron Microscopy, Monash University, VIC 3800, Australia

${ }^{3}$ Department of Materials Science and Engineering, Monash University, VIC 3800, Australia

Corresponding Author

Nagarajan Valanoor* nagarajan@unsw.edu.au 
Figures $\mathrm{S} 1$ indeed confirms the domain matching epitaxy (DME) model with long-range lattice registry for $\mathrm{NiO}$ and STO. The HAADF-STEM image from [110] of STO at the interface, which according to DME model leaves only $0.8 \%$ strain.

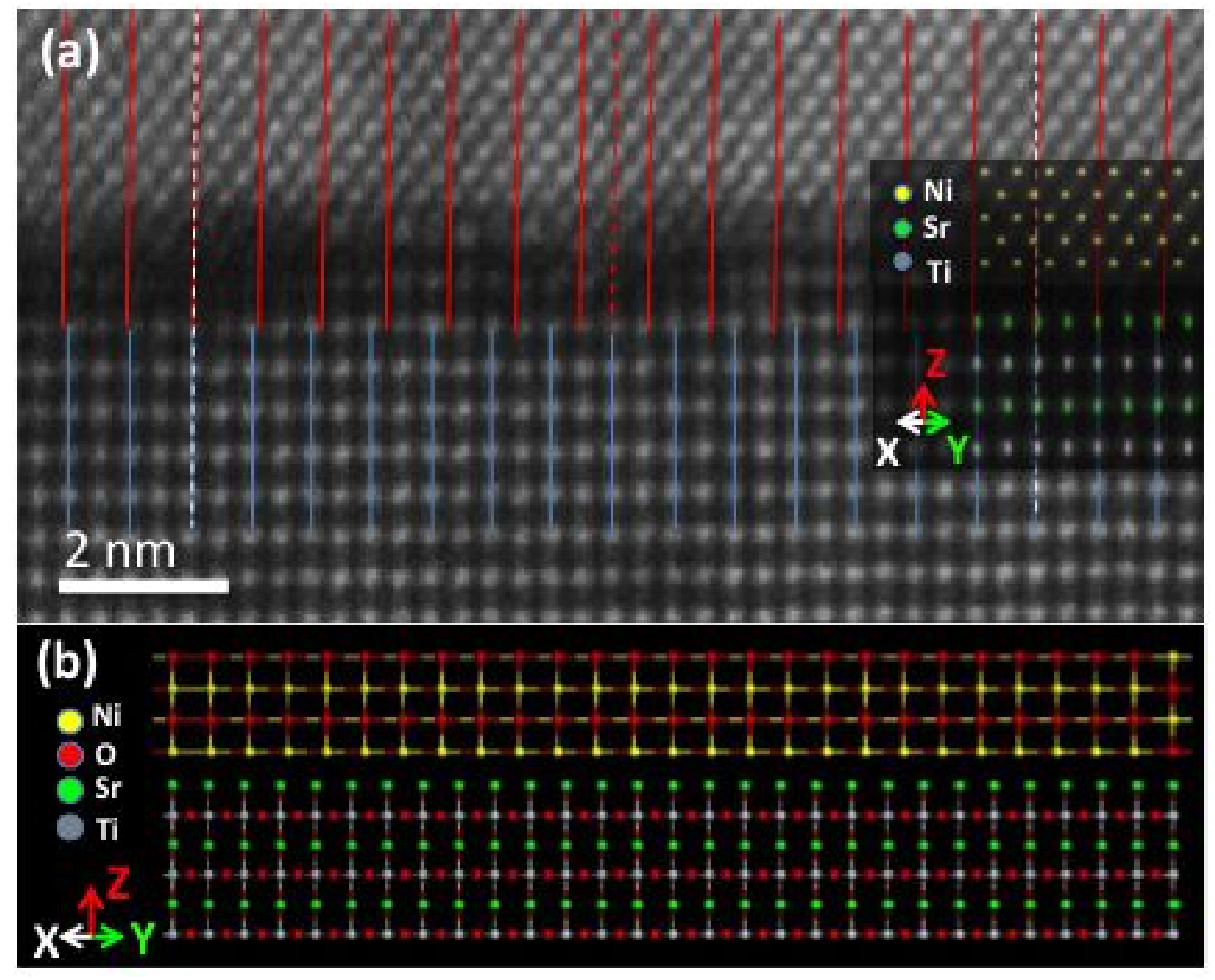

Figure S1 At the interface of annealed NiO nanoisland and STO (with specimen

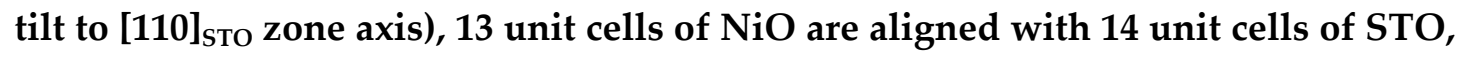
as shown in HAADF-STEM image (a) and schematic in (b). 

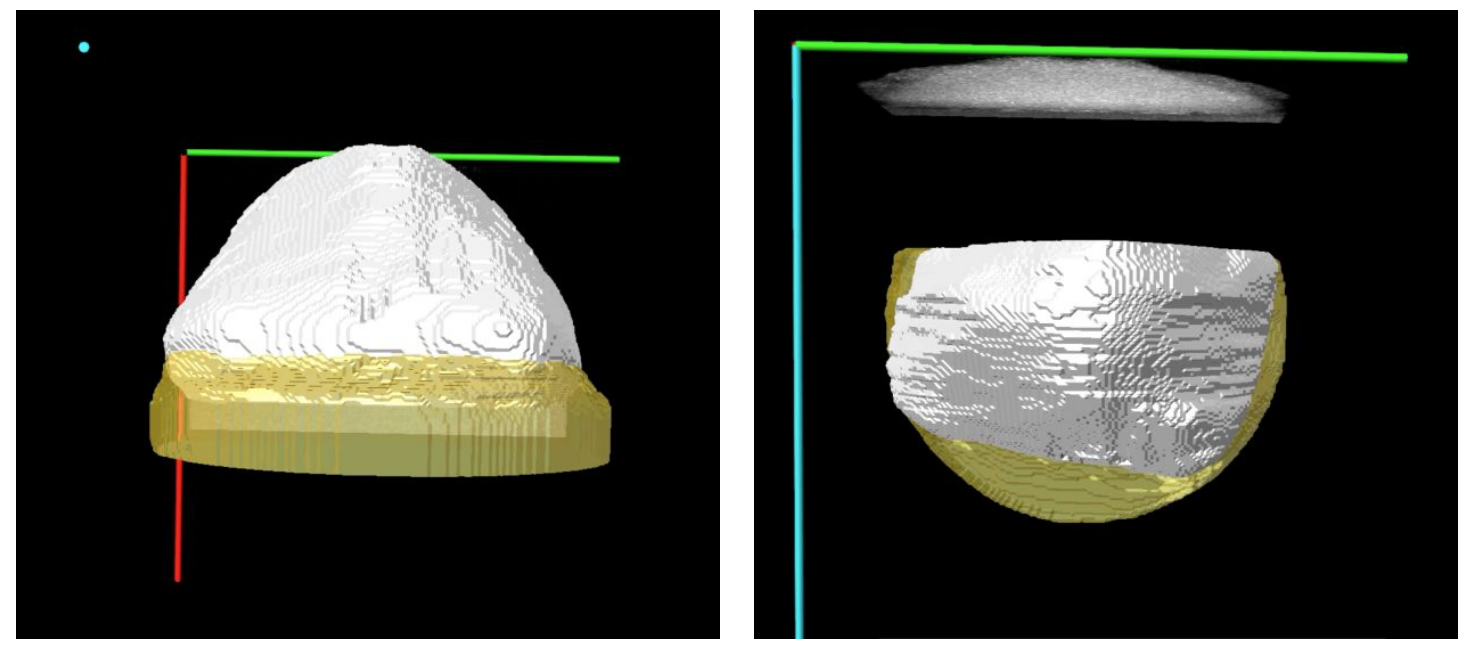

Figure S2. The side view (left) and the top view (right) of a surface rendered representation of the segmented tomographic reconstructed as grown $\mathrm{NiO}$ nanoisland on STO substrate. The top view image shows that the back of this nanoparticle is sectioned off during TEM specimen preparation. Full rotation can be viewed via the video provided. 